\title{
Urinary Biomarkers in Lupus Nephritis
}

\author{
Joyce Reyes-Thomas, Irene Blanco, and Chaim Putterman \\ Division of Rheumatology, Albert Einstein College of Medicine, Forchheimer Building, Room \\ 701N, 1300 Morris Park Ave, Bronx, New York, NY 10461, USA
}

Chaim Putterman: chaim.putterman@einstein.yu.edu

\begin{abstract}
Renal involvement in patients with systemic lupus erythematosus in the form of severe lupus nephritis is associated with a significant burden of morbidity and mortality. Conventional laboratory biomarkers in current use have not been very successful in anticipating disease flares, predicting renal histology, or decreasing unwanted outcomes. Since early treatment is associated with improved clinical results, it is thus essential to identify new biomarkers with substantial predictive power to reduce the serious sequelae of this difficult to control lupus manifestation. Indeed, considerable efforts and progress have been made over the last few years in the search for novel biomarkers. Since urinary biomarkers are more easily obtainable with much less risk to the patient than repeat renal biopsies, and these may more accurately discern between renal disease and other organ manifestations than their serum counterparts, there has been tremendous interest in studying new candidate urine biomarkers. Below, we review several promising urinary biomarkers under investigation, including total proteinuria and microalbuminuria, urinary proteomic signatures, and the individual inflammatory mediators interleukin-6, vascular cell adhesion molecule-1, CXCL16, IP-10, and tumor necrosis factor-like weak inducer of apoptosis.
\end{abstract}

\section{Keywords}

Biomarkers; Lupus nephritis; Urinary biomarkers; Proteomics; TWEAK; SLE

\section{Introduction}

Systemic lupus erythematosus (SLE) is a chronic, potentially life-threatening disease that is characterized by a broad range of clinical manifestations, an often unpredictable temporal sequence of organ involvement, and disease flares that may cause permanent injury. One of the most common manifestations of SLE is lupus nephritis, which can be seen in up to 60\% of all SLE patients [1]. Furthermore, 10-15\% of nephritis patients progress to end-stage renal disease (ESRD) requiring hemodialysis, and the 5-year survival of nephritis patients is stalled at $82 \%$, whereas 5-year survival for those without nephritis is $92 \%$ [1]. Despite the fact that several efficacious therapies have been used to treat lupus nephritis, the incidence of ESRD from lupus nephritis increased during the period of 1982-1985 [2], and from 1996 to 2004 showed no change [3]. This may reflect the limitations of our current treatment options, poor access to health care, late diagnosis, or delay in treatment [3].

Earlier treatment has a beneficial effect on the prognosis of lupus nephritis, and it has been shown that late diagnosis of lupus nephritis is correlated with a higher frequency of renal insufficiency [4]. Moreover, delayed diagnosis is associated with an increased incidence of 
ESRD, again underlining the importance of early diagnosis in this difficult to control disease [5].

Histological analysis of kidney tissue is a valuable tool for diagnosis, assessment, and prognostication in lupus patients. However, kidney biopsy can be accompanied by significant morbidity and, therefore, is not usually performed serially. Furthermore, with an essentially "blind" needle biopsy, there can be a question of how representative are the limited number of glomeruli usually obtained of kidney activity and chronicity. A noninvasive, easily obtainable, and accurate marker that can be followed serially may therefore be of great value in monitoring lupus patients. Laboratory markers in current use, which include serological determination of serum anti-double-stranded (ds)DNA antibodies and complement levels, can be helpful clinically, but the correlation between those and lupus renal disease is imperfect. Sensitivity and specificity for active lupus nephritis among all SLE patients diverge according to different studies and tests used (enzyme immunoassay vs. immunofluorescence) and are depicted in Table 1 [6-8].

It is essential to note that these sensitivities and specificities also varied according to the gold standard used. Esdaile et al. evaluated these markers as predictors 3, 6, and 9 months prior to a renal flare as determined by the renal components of the Systemic Lupus Erythematosus Disease Activity Index (SLEDAI) [8], while Moroni et al. looked more specifically at sensitivity and specificity of detecting nephritic and proteinuric flares of patients who already carried the diagnosis of lupus nephritis [7]. In the prospective longitudinal study by Moroni et al., anti-dsDNA, anti-C1q, C3, and C4 all had poor positive predictive values (ranging from $28 \%$ to $38 \%$ ). Although the best multivariate analysis model for renal flare prediction was obtained by combining anti-C1q with $\mathrm{C} 3$ and $\mathrm{C} 4$, their data clearly showed that anti-C1q antibodies were less reliable in predicting flares in nonproliferative nephritis and flares in the presence of anti-phospholipid antibodies. Furthermore, none of these traditional markers has been shown to possess the ability to predict histology. Clearly, the lack of specificity of our current markers for lupus nephritis and inability to predict histology highlight the pressing need for a true biomarker for lupus nephritis.

One proposed definition of a biomarker is a "cellular, biochemical, molecular, or genetic alteration by which a normal or abnormal biologic process can be recognized and/or monitored and that may have diagnostic or prognostic utility" [9]. Reliable biomarkers may assist in precise evaluation of disease activity, identify patients at risk for organ damage or recurrent flares, and facilitate early and accurate evaluation of responses to treatment [10]. Required criteria for a given measured parameter to serve as a biomarker have been proposed and include the following: (1) it is biologically plausible and relevant to disease pathogenesis, (2) it should accurately reflect changes in disease activity, and (3) it is simple to measure for routine use [10]. However, despite tremendous strides in our understanding of the pathogenesis of SLE, intense interest, and an urgent need for the development of such biomarkers, none has been proven to date to truly reflect disease activity, predict the risk of future organ involvement, response to treatment, or prognosis [9-13].

While we still do not possess a validated biomarker for lupus nephritis, the search is visibly underway. Although the traditional serum biomarkers have not proved to be an appropriate gauge of lupus nephritis, there have been major advances in the discovery of potential serum biomarkers, which are reviewed in separate articles within this issue. Since SLE is a systemic disease, serum biomarkers may indeed be appropriate monitoring and diagnostic tools for systemic disease activity. With respect to lupus nephritis, however, urinary biomarkers may be more specific for kidney damage than serum biomarkers, particularly in SLE patients with active systemic disease. Furthermore, obtaining urine for laboratory 
testing is much easier and less invasive, making urine a more ideal biological sample for a disease that requires repetitive screening.

In this article, we will review several potential urinary biomarkers for lupus nephritis, beginning with the historical urinary biomarker - total proteinuria. We will then expound upon several prospective biomarkers, including microalbuminuria, and novel approaches using proteomic signatures in both the adult and pediatric populations. Subsequently, we will discuss several urinary cytokines, chemokines, and adhesion molecules individually, including interleukin (IL)-6, vascular cell adhesion molecule (VCAM)-1, CXCL16, and IP-10. Finally, we will discuss in some detail a novel tumor necrosis factor (TNF) superfamily member, TNF-like weak inducer of apoptosis (TWEAK), and its role as a potential urinary biomarker for lupus nephritis.

\section{Proteinuria}

The hallmark of lupus glomerulonephritis is proteinuria and, at the present time, it is the principal urinary biomarker that is measured when screening for the disease [1,14]. In fact, it is used in several validated scoring systems to measure disease activity, including the SLEDAI and the British Isles Lupus Assessment Group index [15,16]. Currently, however, there are no set recommendations as to how to screen for elevated urinary protein levels in lupus patients. Moreover, there are several methods used for analysis of urinary protein, as depicted in Table 2, which are further discussed in the following section. Given then the lack of guidelines and multiple techniques for analysis, clinicians vary greatly in their screening for proteinuria. In a recent survey of American rheumatologists, $65 \%$ reported using a urine dipstick to screen for proteinuria, while $17 \%$ used a spot (untimed) protein to creatinine ratio, and only $12 \%$ of those surveyed used a timed $24-\mathrm{h}$ collection to screen for proteinuria [17]. Despite its pervasive use, however, the dipstick has shown to be an inaccurate tool in the assessment of proteinuria where the gold standard is either a 24-h urine protein collection, or for an inadequate collection, a $24-\mathrm{h}$ protein to creatinine ratio $[18,19]$.

Nevertheless, the 24-h urine collection is a cumbersome and often impractical tool, leading to the investigation of spot measures. In diseases such as diabetic nephropathy, the spot urinary protein to creatinine ratio has been shown to correlate well with 24-h protein to creatinine ratios [20,21]. Indeed, the National Kidney Foundation currently recommends spot protein to creatinine ratios for the assessment of chronic kidney disease over timed urine collections [22-24]. With this in mind, the ACR Renal Disease Subcommittee recommends measuring a morning void protein to creatinine ratio, though a spot urine is acceptable as well, as a screening tool for proteinuria in nephritic patients [25]. There are no separate recommendations for the initial screening of patients who have never carried the diagnosis of nephritis vs. patients with biopsy-proven nephritis.

However, this recommendation is not without controversy. There have been conflicting reports with regards to the reliability of the spot protein to creatinine ratio in nephritis flares. Leung et al. previously reported that the spot ratio is a good alternative to the 24-h collection [26]. In their study, they looked at 129 paired 24-h and spot urine collections to evaluate if the two were correlated. Their data showed that in fact there was a strong correlation between the spot and 24-h urine protein to creatinine ratio $(r=0.91, p<0.001$.) However, neither the 24-h nor the spot ratio was correlated with the SLEDAI-2K [26].

In contrast, Birmingham et al. subsequently found that in fact, the spot urine protein to creatinine ratio is an unreliable estimate of 24-h proteinuria [27]. In their study, they analyzed paired spot urines and 24-h urine collections from 64 patients with biopsy-proven lupus nephritis. They found that over the entire range of proteinuria, there was strong correlation between the spot and 24-h urine collection with a fair concordance correlation 
coefficient or $\mathrm{CCC}(r=0.778, \mathrm{CCC}=0.764)$. However, while a positive correlation was present over the protein to creatinine ratio that is typically seen in nephritis patients $(0.5-$ 3.0), the concordance was poor $(r=0.529, \mathrm{CCC}=0.479)$ [27]. They attributed this discrepancy in results to the fact that the correlation coefficient is sensitive to a wide range of measurements. Therefore, when analyzed over a narrow range of values for proteinuria, the test was no longer reliable. Recently, Hebert et al. revisited the data from Leung's original article [28]. Twenty-four-hour protein to creatinine ratios were imputed based on the published data, and the analysis was repeated to specifically look at proteinuria in the sub-nephrotic rage of 0.5-3.0 typically seen in lupus nephritis patients. Now, the data showed that indeed, the spot protein to creatinine ratio was not a reliable estimate for the 24$\mathrm{h}$ urine collection [28].

Of the American rheumatologists surveyed with regards to screening practices, $4 \%$ stated that they screened for proteinuria using microalbuminuria assays [17]. Microalbuminuria, which is defined as the "abnormally increased excretion rate of albumin in the urine in the range of 30-299 $\mathrm{mg} / \mathrm{g}$ creatinine" [29], is classically used to detect diabetic glomerulonephropathy in cases of normal range proteinuria [22-24]. This technique, therefore, has potential use to detect early nephritis in lupus patients with low levels of proteinuria who may in fact have significant disease on biopsy [30]. However, studies investigating the use of screening for microalbuminuria in lupus patients have yielded mixed results. Despite the fact that lupus patients without renal disease may present with microalbuminuria, screening has not been able to predict biopsy results or the subsequent development of nephritis [31,32]. One possible role for the measurement of microalbuminuria is post-induction therapy for nephritis, where levels $<0.5 \mathrm{~g} /$ day are associated with improvement on repeat renal biopsy [33]. In lieu of measuring only microalbuminuria in lupus nephritis patients, the microalbumin to protein ratio may be of use in patients with normal urinary protein levels where increases in this ratio may be more sensitive in identifying nephritis patients at risk for a flare [34].

Even though the spot albumin to creatinine ratio is correlated with 24-h urine albumin collection, it has not been thoroughly studied in SLE [35]. Presently, it is unknown if the spot albumin to creatinine ratio will be a reliable method to screen lupus patients for proteinuria and early kidney disease as it is in diabetic patients [22-24]. Finally, a new method recently proposed in the screening for proteinuria is the use of short-interval timed urine collections. In this study, a 12-h overnight urine had a very good correlation with the 24-h urine protein to creatinine ratio and is a much more convenient protocol for patients to follow [36]. While promising, this is a new regimen and will need further investigation before it can be accepted as a standard screening practice.

\section{Urine proteomics}

While total urine protein remains a commonly used (albeit imperfect) marker for nephritis, more reflective than predictive of disease activity, and microalbuminuria has not yet been established by large studies to be an accurate biomarker, investigators are still searching for an appropriate urinary biomarker by analyzing the various other proteins in the urine of SLE patients.

Traditionally, the separation and analysis of proteins in biological fluids required labor, material, and time-intensive two-dimensional gel electrophoresis. As an alternative, liquid chromatography-tandem mass spectrometry (LC/MS/MS) has been used as a relatively less time-consuming method with higher sensitivity for smaller proteins. With the advent of surface-enhanced laser desorption/ionization time-of-flight mass spectrometry (SELDI-TOF MS), however, the number of studies involving urinary protein profiles associated with 
lupus nephritis have increased substantially, particularly because of the ability to perform rapid profiling of multiple urine samples, the relatively low amounts of urine needed for analysis $(5-10 \mathrm{uL})$, and the possibility for detection of low-molecular-weight proteins, especially below $20,000 \mathrm{Da}[13,37,38]$. The main drawback for SELDI-TOF is that it identifies patterns of protein expression but cannot identify specific proteins [38]. By integrating on-chip protein sequencing or LC/MS/MS, however, proteins may be subsequently identified after initial screening with SELDI-TOF MS [39]. The advancement of protein profiling techniques has yielded substantial insight into the human urine proteome, and in this section, we will review the most recently studied proteins within the urinary proteome of patients with lupus nephritis.

\section{Urinary proteomics in pediatric lupus nephritis}

Using SELDI-TOF MS, Suzuki et al. reported a urinary proteomic signature for SLE nephritis in the pediatric population consisting of eight biomarker proteins of varying weights [40]. A total of 32 SLE patients were included into the study, with 11 juvenile idiopathic arthritis (JIA) patients used as controls. Using this technique, they consistently found mass spectrometry peaks with mass-to-charge ratios of 2.763, 22, 23, 44, 56, 79, 100, and 133 in patients with class III, IV, or V nephritis. There were no statistically significant differences between the proteomic signatures of the controls (JIA patients) and the SLE patients without nephritis. Although a trend was observed, there was no significant difference between the proteomic signatures of class III and IV vs. class V. There was, however, a strong correlation of this proteomic signature with renal disease activity and a moderate correlation with renal damage [40].

Subsequently, using surface-enhanced or matrix-assisted laser desorption/ionization time-offlight mass spectrometry (MALDI-TOF MS), Suzuki et al. were able to identify the individual proteins in the urinary protein signature of pediatric lupus nephritis: transferrin, ceruloplasmin, $\alpha 1$-acid-glycoprotein (AGP), lipocalin-type prostaglandin D-synthetase (LPDGS), albumin, and albumin-related fragments [41]. They further correlated these proteins with disease activity in a study with 98 predominantly African-American SLE patients, compared to 30 control patients with JIA. Urinary and blood samples were taken every 3 months for up to 18 months, and levels of transferrin, ceruloplasmin, AGP, and L-PDGS were measured. At baseline, urinary levels of all four proteins were significantly higher in children with SLE than those with JIA, but plasma levels of these proteins were comparable between the two groups with the exception of plasma transferrin, which was significantly higher in the JIA group. Urinary levels of protein excretion standardized by urine creatinine for all four proteins were significantly greater in the group with active lupus nephritis compared to those with SLE with no nephritis or inactive nephritis, and also compared to the JIA controls. Importantly, urinary levels of transferrin, AGP, and L-PDGS significantly increased at least 3 months prior to the clinical diagnosis of worsening lupus nephritis. Moreover, none of the traditional biomarkers (protein to creatinine ratio, creatinine clearance, and $\mathrm{C} 3$ and $\mathrm{C} 4$ levels) was able to predict lupus nephritis flares, implying that transferrin, AGP, and L-PGDS are, in fact, superior biomarkers for lupus nephritis in predicting disease flares.

Unfortunately, this study by Suzuki et al. did not have enough subjects to correlate the different proteins to the histological type of nephritis. In addition, AGP and L-PGDS were both found to be good predictors of diabetic renal disease in the adult population [42], implying that in adults, it may not be very specific for lupus nephritis in patients with other comorbidities. This may not pose a significant hurdle, however, as there are already validated and known screening tools for diabetes, and the diabetic and lupus populations are quite discernable through other clinical criteria. 
In summary, initial indications are that transferrin, AGP, and L-PDGS may serve as potential biomarkers for impending nephritis flares in pediatric lupus, and additional studies are warranted.

\section{Urinary proteomics in adult lupus nephritis}

The use of SELDI-TOF MS has also yielded important proteomic discoveries in the adult lupus nephritis population. In 2006, Mosley et al. identified two proteins by the use of SELDI-TOF MS, which yielded a sensitivity and specificity for active lupus nephritis of 92\% each [43]. Using MALDI-TOF MS and an artificial neural network analysis, Varghese et al. were able to identify 11 urinary proteins associated with focal segmental glomerulosclerosis, SLE classes III, IV, and V, as well as membranous nephritis and diabetic nephropathy [44]. Moreover, they were able to differentiate lupus nephritis from focal segmental glomerulosclerosis, membranous nephropathy, and diabetic nephropathy based on the signature of these 11 proteins with a sensitivity of $86 \%$ and a specificity of $89 \%$. The proteins included in this proteomic signature include AGP, transferrin, $\alpha-1$ microglobulin, zinc $\alpha$-2-glycoprotein, $\alpha$-1-antitrypsin, complement factor $\mathrm{B}$, haptoglobin, transthyretin, plasma retinol binding protein, albumin, and hemopexin. However, only 32 patients in total were studied, 11 of whom had lupus nephritis. Of note, the same group identified a set of urine proteins that could differentiate between the different classes of lupus nephritis; however, they were unable to replicate these results in a subsequent cohort of patients [45].

Zhang et al. further expanded upon the known urinary proteome of lupus nephritis in 2008 using SELDI-TOF MS to screen serial urine samples from 25 moderate to severe SLE nephritis flares from 19 patients with known class III $(n=5)$, class IV $(n=11)$, or class V $(n=3)$ lupus nephritis [13]. They identified several proteins which showed statistically significant variation in accordance to flare cycles and then further identified these proteins using on-chip peptide sequencing as hepcidin (both 20 and 25 amino acid isoforms), $\alpha-1$ antitrypsin, and a fragment of albumin. Hepcidin 20 was found to increase 4 months prior to flare, while hepcidin 25 was found to decrease at flare and return to baseline 4 months after flare. $\alpha$-1-antitrypsin increased at flare, whereas the albumin fragment ( $\mathrm{N}$-terminal region) increased 4 months pre-flare compared to baseline. Interestingly, hepcidin is regulated by IL-6 and TNF, both cytokines that are involved in the development of lupus nephritis [46]. Since hepcidin 20 expression increased 4 months prior to flare and hepcidin 25 seemed to return to baseline 4 months after a flare resolved, the authors of this study postulated that hepcidin 20 could possibly be used to predict flares and hepcidin 25 may be a potential marker for response to therapy.

While SELDI-TOF MS and integrated techniques have certainly shed light upon the urinary proteome, investigators have also made substantial discoveries using the traditional, laborintensive 2D-gel electrophoresis. In a recent study by $\mathrm{Wu}$ et al., 71 different proteins were isolated from the urine of mice with induced nephritis via 2D-gel electrophoresis. In this study, C57BL/6 (B6) mice were injected with pre-formed anti-glomerular basement membrane (anti-GBM) antibodies to induce nephritis, a well-established technique and reproducible model for lupus nephritis. Wu et al. then proceeded to validate four of those proteins: PGDS, renin or total protease, serum amyloid-P (SAP), and superoxide dismutase (SOD) [47].

Levels of PGDS were confirmed to be elevated in the urine, and urinary levels of its enzymatic product, PGD2, as well as SOD, total protease, and SAP all increased after the induction of nephritis, and all correlated with renal pathology. Urinary levels of all four molecules were also increased in two other established murine lupus models, MRL.lpr and B6.Sle1.Sle3 mice, compared to controls. In B6.Sle1.Sle3 mice, urinary levels of PGDS, 
SOD, total protease, and SAP also increased with age and disease, each correlating well with one another. The four markers were also superior to the traditional measures of 24-h proteinuria and BUN with respect to predictive ability and correlation with glomerulonephritis scores and pathology activity indices, with total protease having the strongest correlation [47].

\section{Urinary cytokines, chemokines, and adhesion molecules}

\section{IL-6}

IL-6 is a pleiotropic cytokine expressed by antigen-presenting cells such as macrophages, Bcells, and dendritic cells and is also produced by fibroblasts, mesangial cells, T cells, endothelial cells, epithelial cells, keratinocytes, and astrocytes [48]. Production of IL-6 is induced by IL-1, TNF, lipopolysaccharide, and platelet-derived growth factor [48]. IL-6 induces B-cell differentiation into antibody-producing cells, T-cell differentiation into effector cells, and also induces the production of many acute phase proteins [49-52]. Richards et al. initially showed that IL-6-deficient BALB/c mice with pristine-induced lupus had lower titers of anti-dsDNA antibodies when compared to IL-6 intact mice, suggesting a possible role for IL-6 in the pathogenesis of lupus nephritis and disease flares [53].

Because of its purported role in the pathogenesis of lupus nephritis, urinary IL-6 has been a potential biomarker of interest. Urinary levels of IL-6 in 29 patients with active lupus nephritis were measured, and patients with WHO class IV nephritis on renal biopsy were found to have significantly higher IL-6 levels than other classes of nephritis $(p<0.01)$ [54]. Perhaps because patients with class IV nephritis seemed to have higher urinary excretion of IL-6, a subsequent study with only six SLE patients (5/6 with class III nephritis and only 1/6 with class IV nephritis) found no significant increase in urinary IL-6 when compared to controls [55]. In a subsequent study in 2000, Tsai et al. examined the urine of 44 patients (15 active lupus nephritis, 12 inactive lupus nephritis, and 17 normal controls) and measured amounts of $\beta 2$-microglobulin, Tamm-Horsfall glycoprotein, IL-2, IL-6, and IL-8. Of the active lupus nephritis patients, 11 had class IV lupus nephritis, and four had class III. IL-6 urinary excretion was found to be significantly higher in patients with active nephritis compared to inactive nephritis $(p=0.034)$ and compared to controls $(p<0.001)$. In inactive nephritis, urinary IL-6 levels were also higher than in controls $(p<0.001)$. IL-8 also showed a similar pattern. In addition, both IL-8 and IL-6 urinary levels decreased in 5/6 patients with active lupus nephritis after receiving two courses of cyclophosphamide within 6 months. The only patient who did not exhibit lower urinary IL-6 and IL-8 levels subsequently developed neurological symptoms and was deemed refractory to immunosuppressive therapy—-suggesting that IL- 6 could be a good biomarker for both disease activity and response to treatment [56]. However, while the preliminary data from small trials of urinary IL-6 is promising, a larger non-published cohort of SLE patients (SLE=143, positive $\mathrm{ANA}=128$, normal controls $=73$ ) did not find any significant difference in the urinary IL-6 levels in patients with and without lupus nephritis [49].

VCAM-1

Leukocytes are attracted to the site of injury in nephritis by chemotactic stimuli, and once activated, release proteases and reactive oxygen species contributing to tissue damage and fibrosis [55]. VCAM-1 is a member of the Ig superfamily and acts as a ligand for $\alpha_{4}$ integrins, supporting tethering and adhesion of leukocytes to endothelial cells [57]. Expression of VCAM-1 is regulated by TNF and IL-1, and these latter cytokines have been shown to sequentially induce endothelial VCAM-1 expression in MRL/lpr lupus-prone mice [58]. Moreover, VCAM-1 expression has been shown to be increased in the mesangial cells of mice in a lipopolysaccharide-induced model of lupus nephritis [59]. 
Wu et al. obtained urine from mice with mild and severe anti-GBM-induced nephritis. Using membranes bearing antibodies to 62 different cytokines, chemokines, and receptors and subsequent confirmatory enzyme-linked immunosorbent assays, they found that the urinary levels of VCAM-1, P-selectin, soluble tumor necrosis factor receptor-1, and CXC chemokine ligand 16 (CXCL16) were elevated in the urine of mice with severe nephritis when compared to those with mild nephritis [60]. Wu et al. further studied these molecules in multiple murine lupus strains as well as in human lupus nephritis. They were able to show that urinary VCAM-1 levels correlated well with urine protein in all three of their murine lupus nephritis models (B6.Sle1.lpr, MRL.lpr, and NZM2410 mice). Additionally, the urinary VCAM-1 levels were found to be higher than their corresponding serum levels, and diseased MRL.lpr kidneys exhibited increased VCAM-1 staining in the capillaries, endothelium, glomerular cells, and interstitial inflammatory cells when compared to young mice prior to disease onset [61].

To determine the relevance of these findings in human disease, Wu et al. compared urine samples from 38 SLE patients vs. 15 normal controls and six RA control patients and found that patients with SLE showed significantly higher VCAM-1 levels than the controls.

VCAM-1 levels also correlated with urine protein to creatinine ratios and SLEDAI scores, and SLE patients with active nephritis exhibited the highest levels of VCAM-1 in the urine (Fig. 1) [61]. Similar results were reported in a preliminary study by Kiani et al., who found that urinary VCAM-1 levels were highly correlated with higher renal activity (measured by the Systemic Lupus International Collaborating Clinics Renal Activity Score), hematuria, proteinuria, and pyuria in 81 SLE patients [62]. These results corroborate those of previous smaller studies: in a small study with six lupus nephritis patients and nine healthy controls, urinary VCAM-1 levels were shown to be significantly higher in lupus nephritis vs. normal controls or patients with IgA nephropathy [55]. Similarly, another study $(n=27)$ showed that higher levels of serum VCAM-1 correlated with increased disease activity in humans with active lupus nephritis (WHO class III and IV, $n=11$ ) when compared to patients with inactive or mild lupus nephritis (WHO class I and II, $n=13$ ) [63]. These studies all suggest that VCAM-1 is associated with nephritis activity in SLE patients. However, further studies in regards to long-term renal disease, as well as prospective trials to see if a rising VCAM-1 level can be a good predictor of disease flare, have not yet been carried out.

\section{CXCL16}

CXC chemokine ligand 16 (CXCL16) is a chemokine expressed on dendritic cells and macrophages, with important roles including T-cell and NK-cell recruitment and intracellular adhesion mediated by interactions with its receptor, CXC receptor 6 (CXCR6) $[64,65]$. Besides its role in inflammation, CXCL16 has been implicated in the development of atherosclerosis, phagocytosis of Gram-positive and Gram-negative bacteria, and as an inducer of NF-אB activation [66-68]. More recently, a possible role for CXCL16 in the pathogenesis of lupus nephritis has been explored as well.

Through genome-wide messenger RNA (mRNA) expression analysis using microarray technology, Teramoto et al. found increased expression of CXCL16 in lupus mice (MRL/ lpr) glomeruli. They postulated that in the glomeruli of mice with lupus nephritis, there is production of various chemokines, including CXCL16 and its receptor CXCR6, that stimulate recruitment of TH1 polarized T cells [69]. Furthermore, in the same study discussed above that examined VCAM-1, Wu et al. found that urinary levels of CXCL16 were elevated in the urine of mice with spontaneous lupus nephritis (B6.Sle1.lpr, MRL.lpr, and NZM2410 models) and were higher than the corresponding serum levels of CXCL16 [61]. Moreover, CXCL16 was hyperexpressed in renal tissue of diseased mice, and the urine of SLE patients was also rich in CXCL16, with good correlation to SLEDAI scores.

CXCL16 had an area under the curve of 0.86 in the receiver operation characteristic curve- 
denoting good sensitivity and specificity for distinguishing SLE patients from healthy controls [61]. There have been no further studies of CXCL16 at the time of this review, and additional studies appear warranted.

IP-10

Interferon- $\gamma$-inducible protein 10 (IP-10), also known as CXCL10 [chemokine (C-X-C motif) ligand 10] is a chemokine secreted by interferon- $\gamma$-stimulated endothelial cells, fibroblasts, and monocytes. Together with its receptor, CXC receptor 3 (CXCR3), IP-10 promotes the migration of $\mathrm{T}$ cells to sites of inflammation and is also known to play a role in the downregulation of angiogenesis [70,71]. IP-10 mRNA expression has been shown to be upregulated in the inflamed lungs of MRL/lpr lupus mice, and CXCR3 was concomitantly shown to be significantly elevated in CD4+ T cells, macrophages, and double-negative $\mathrm{T}$ cells migrating to the lungs [72]. Serum levels of IP-10 are increased in patients with SLE and particularly in those patients with lupus nephritis [73].

Given the known pathogenic relevance of IP-10 and the increased levels found in human SLE serum, Avihingsanon et al. studied 26 patients, 14 of whom had class IV nephritis, and 12 of whom had class II, III, V, or VI [74]. They examined the urinary mRNA levels of IP-10, CXCR3, transforming growth factor- $\beta$ (TGF- $\beta$ ), and vascular endothelial growth factor and found that levels of all four mRNAs were increased in class IV lupus nephritis patients when compared to other classes of lupus nephritis. The receiver operation characteristic curve for log-IP-10 urinary mRNA levels had an area under the curve of 0.89 compared to an area under the curve of 0.55 for 24-h urinary protein, indicating that urinary IP-10 mRNA is a significantly better test for class IV lupus nephritis. Furthermore, patients who responded to therapy had significantly lower levels of IP-10, suggesting that IP-10 can be used as a barometer for treatment efficacy [74].

IP-10 as a biomarker shows promise in its diagnostic capabilities as it appears to be quite specific for class IV lupus nephritis and, if this observation is validated, could potentially be used in lieu of kidney biopsy to dictate treatment or change in management. Undoubtedly, further studies are necessary to validate this potentially useful candidate biomarker.

\section{TWEAK}

The cytokine TWEAK was first discovered in 1997 [75] and assigned to the TNF superfamily based on characteristic sequence motifs. TWEAK is synthesized as a 249 amino acid type II transmembrane protein. Similar to TNF, TWEAK is processed via a furin cleavage site in the extracellular domain into a soluble form (the C-terminus extracellular receptor binding domain) that circulates as a trimer, believed to be the primary mediator of the biological effects of the molecule. The major source of circulating TWEAK is believed to be activated monocytes and macrophages.

The TWEAK receptor (TWEAK-R), a TNF receptor superfamily member more commonly known as Fn14, is thought to be the primary TWEAK receptor. Similarly, TWEAK is the only known TNF family member to bind to Fn 14 . The cytoplasmic tail of Fn14 contains a binding site for the TNF receptor-associated factors 1,2,3, and 5, adapter proteins that activate downstream signaling. Fn14 is expressed on endothelial cells, vascular smooth muscle cells, kidney, heart, lung, spleen, brain, monocytes/macrophages, and NK cells, but not B or T cells [76,77], and is upregulated under conditions of tissue stress and inflammation $[78,79]$.

In kidney cells, TWEAK mediates important biological effects, including modulation of cell survival and upregulation of proinflammatory mediators [80]. In human mesangial cells, podocytes, and tubular cells, which we found all express Fn14, TWEAK induces the 
expression of multiple inflammatory mediators, including RANTES, monocyte chemoattractant protein (MCP)-1, IP-10, MIP-1 $\alpha$, ICAM-1, VCAM-1, MMP-1, and MMP-9 [81]. Importantly, conditioned media from TWEAK-stimulated mesangial cells induced significant migration of peripheral blood mononuclear cells in chemotaxis assays, including lymphocytes and macrophages. Furthermore, we found that TWEAK significantly stimulated proliferation of mesangial cells and podocytes [82]. Finally, kidney biopsies of several lupus nephritis patients demonstrated strong glomerular and tubulointerstitial staining for Fn14.

To confirm the importance of TWEAK in the pathogenesis of lupus nephritis, we analyzed the effects of Fn14 deficiency or TWEAK blockade on severity of disease in chronic graft vs. host (cGVH)-induced lupus in B6 mice [83]. In this model, a single injection of $10^{8}$ MHC II-incompatible splenocytes to unirradiated mice induces within 2 to 4 weeks autoantibodies and renal disease characteristic of lupus [84,85]. We found that titers of antidsDNA and anti-chromatin antibodies were no different between B6 Fn14 knockout and wild type mice injected with alloreactive splenocytes. However, kidney disease (as assessed by proteinuria) was significantly less severe in Fn14 knockout mice, as was kidney IL-6, MCP-1, and RANTES staining and macrophage infiltration. Similarly, we found that B6 Fn14 wild type mice with cGVH-induced lupus treated with a monoclonal anti-TWEAK antibody had significantly less proteinuria, as well as decreased kidney expression of IL-6, MCP-1, RANTES, and KC than control treated mice [86].

Interesting studies by Kaplan et al. provide some direct support for a possible role of TWEAK in the etiopathogenesis of human SLE as well, via induction of monocyte apoptosis mediated by high TWEAK expression on T cells [87,88]. Kaplan et al. suggest that increased monocyte killing may have a double contribution to lupus pathogenesis: increasing the load of nuclear autoantigens released by apoptotic cells, while concurrently decreasing the clearance of apoptotic debris [87].

We had found that murine and human kidney cells express Fn14 and respond to TWEAK stimulation by upregulating the production of cytokines/chemokines instrumental in the pathogenesis of lupus nephritis [80,81]. Moreover, TWEAK induces glomerular cell proliferation and promotes chemotaxis of macrophages and lymphocytes, in vitro and in vivo. Furthermore, we demonstrated a role for TWEAK/Fn14 interactions in the pathogenesis of nephritis in a lupus animal model $[81,86]$. Based on these considerations, we hypothesized that urinary TWEAK (uTWEAK) levels (as a proximal cause of chemokine induction) might also be useful as a biomarker in human lupus patients with nephritis. Indeed, uTWEAK may be even more revealing than individual chemokines, since TWEAK is proximal in the inflammatory cascade and induces several nephritis-related mediators.

We then examined uTWEAK levels in samples from the Ohio Lupus Study (Columbus, OH, USA) and Montefiore Medical Center (Bronx, New York, NY, USA), and found that uTWEAK levels were significantly higher in patients with active nephritis (score on the renal components of the SLEDAI scale, or rSLEDAI, of $\geq 4$ ) compared to never or nonactive nephritis (rSLEDAI=0; 16.3(9.9-23) median (interquartile range) vs. 5.5(2.3-16.8) $\mathrm{pg} / \mathrm{mg}$ creatinine, $p<0.001, n=78$ ) [89]. Moreover, we found a significant correlation between uTWEAK and rSLEDAI scores, indicating a linear relationship between the levels of uTWEAK and the extent of nephritis activity. UTWEAK levels were significantly higher in patients with disease relapse $(11.1(8.1-18.2))$ as compared to patients with chronic stable disease (5.2(2.3-15.3) $\mathrm{pg} / \mathrm{mg}$ creatinine; $n=49)$. In the more limited subgroup of lupus patients with previous renal involvement, higher levels of uTWEAK were found during renal relapse (12.4(9.1-18.2)), as compared to chronic stable renal disease (7.6(3.8-14.4) 
$\mathrm{pg} / \mathrm{mg}$ creatinine; $n=35 ; p=0.056$ ). There was a significant correlation between uTWEAK and uMCP-1 levels $(n=51 ;$ rho $=0.5, p<0.001)$. However, we did not find a significant correlation between urinary protein concentrations and TWEAK levels, indicating that increased TWEAK is not reflective of non-specific spillage into the urine with the loss of the glomerular filtration barrier to protein. Furthermore, urine levels of IL-8 and urokinase plasminogen activator receptor were independent of the presence of a disease flare, indicating that the observed increase in UTWEAK levels with nephritis is indeed significant. When patients undergoing a flare were considered separately $(n=31)$, we found that renal relapses were associated with significantly higher uTWEAK levels than non-renal relapses (12.4(9.1-18.2) vs. 5.2(3.0-11.9) $\mathrm{pg} / \mathrm{mg}$ creatinine, $p=0.03)$. Interestingly, patients at the time of a non-renal flare had similar TWEAK levels to patients with chronic stable disease without a flare. This signifies that uTWEAK levels may primarily reflect local disease activity in the kidney, rather than systemic disease [89].

To provide additional support for UTWEAK as a lupus biomarker, we recently completed an analysis of uTWEAK levels cross-sectionally in a new cohort of SLE patients $(n=79)$, with and without biopsy-proven nephritis [90]. Several control populations (SLE patients without nephritis, rheumatoid arthritis patients, osteoarthritis patients, non-lupus renal disease patients, and healthy controls) were analyzed for comparison. We also performed a longitudinal analysis of prospectively followed lupus nephritis patients to examine the ability of TWEAK to predict, or at least reflect, disease flare and remission. Once again, we found that patients with lupus nephritis had significantly higher levels of uTWEAK than those lupus patients without nephritis (13.0 (5.8-30.2) vs. 6.1(1.8-12.81) $\mathrm{pg} / \mathrm{ml}$, respectively, $p=0.039)$. uTWEAK levels did not differ significantly whether a diagnosis of nephritis was made by renal biopsy or on clinical (laboratory) grounds alone. Furthermore, uTWEAK levels were linearly associated with the severity of renal disease, as measured by the rSLEDAI score (Fig. 2). uTWEAK levels in LN patients were also significantly higher than in normals and patients with rheumatoid arthritis. Renal disease itself did not increase uTWEAK levels, since uTWEAK levels in patients with non-lupus renal disease did not significantly differ from other control groups. Furthermore, we compared uTWEAK levels in lupus patients having similar total SLEDAI scores, with and without nephritis. uTWEAK levels were significantly higher in the former group, further indicating that the high uTWEAK levels in lupus nephritis patients is specific for nephritis, rather than reflecting systemic disease activity. Interestingly, in patients with nephritis, there was no correlation between serum and urine TWEAK levels, indicating that uTWEAK is not passively secreted by diseased kidneys but rather is indicative of local production [90].

To establish a possible role of a novel biomarker, it is important to compare its performance to laboratory measures currently in use. To this end, we directly compared the characteristics of uTWEAK as a diagnostic marker to that of serum anti-dsDNA antibody titers and C3 and C4 levels [90]. Using receiver operation characteristic curves to determine how well does each biomarker differentiate between SLE patients with and without nephritis, we found that uTWEAK performed significantly better (area under the curve of $0.72, p<0.001$ ) than the traditional biomarkers of anti-dsDNA antibodies, $\mathrm{C} 3$, and $\mathrm{C} 4(0.6 ; p=0.15,0.58 ; p=0.26$, and $0.63 ; p=0.053$, respectively). In this study, high uTWEAK levels had $50 \%$ sensitivity and $90 \%$ specificity in establishing a non-invasive diagnosis of nephritis in lupus patients. Furthermore, in a simple logistic regression model, when age, sex, and race were adjusted for, lupus patients with high vs. low uTWEAK levels had an odds ratio of $7.4(p=0.001)$ in predicting the presence of nephritis. Importantly, the performance of uTWEAK levels alone exceeded that of serum TWEAK or of the urine/serum TWEAK ratio [90].

To begin to determine the utility of uTWEAK to help monitor renal disease activity over time, we analyzed uTWEAK levels in 13 patients who provided urine samples every 2 
months for 6 months before and after a documented renal flare [90]. uTWEAK levels significantly increased as patients came closer to the time of flare, peaking at the time of flare diagnosis. Following the flare and with treatment, uTWEAK levels fell to baseline within 4 months. The values of uTWEAK at the time of flare were significantly higher than those observed at -4 and -6 months (before the flare), and at +4 and +6 months (after the flare). Combining the results from these 13 patients with an additional group of 31 unselected lupus patients for which serial uTWEAK levels were available (only some of which displayed a renal flare during the course of the follow-up), a statistically significant association was confirmed between uTWEAK levels and the renal disease activity over time [90]. Taken together, the preliminary longitudinal data suggests that uTWEAK levels may not only have value when assessed concurrent with a flare, but when these levels are followed over time, they may predict subsequent flares or response to treatment.

\section{Summary}

Clearly, there is a pressing need in patients with lupus nephritis to find an adequate biomarker predictive of disease activity, histological and prognostic stratification, and response to treatment. Our review is by no means comprehensive; other potential urinary biomarkers, including neutrophil gelatinase-associated lipocalin (NGAL), MCP-1, and several others have been recently reviewed in previous publications [91,92], so these were not the focus of our review. Furthermore, more studies need to be done to look at these novel biomarkers in combination. For example, a pilot study of urine from 40 lupus nephritis patients evaluating transferrin, ceruloplasmin, AGP, L-PDGS, and NGAL revealed that certain proteins correlated with different histological features on biopsy. The preliminary results suggest that these biomarkers may be part of a panel that in combination may eventually be able to predict histology without the need of an invasive biopsy [93].

The sampling of molecules reviewed in this article exemplify the intense interest to find a true biomarker for lupus nephritis. A summary of the potential biomarkers reviewed is depicted in Table 3, which shows that many of these proteins not only correlate with disease activity and damage but may also predict flares, response to treatment, and histology. Although most of the studies are preliminary, their early results are encouraging. We are optimistic that a biomarker will emerge with the ability to positively affect management and decrease the morbidity and mortality of this difficult to control disease manifestation. Currently, however, none has yet been proven with large longitudinal controlled patient studies. At this point, perhaps it may be preferable to channel resources towards clinical studies determining the predictive power of these known molecules in large longitudinal multi-center lupus cohorts, rather than continuing to search for new molecules. Only then will we discover if these promising biomarkers actually alter patient outcomes and improve the lives of the patients with this life-threatening disease complication of SLE.

\section{References}

1. Cameron JS. Lupus nephritis. J Am Soc Nephrol. 1999; 10:413-424. [PubMed: 10215343]

2. Ward MM. Changes in the incidence of end-stage renal disease due to lupus nephritis, 1982-1995. Arch Intern Med. 2000; 160:3136-3140. [PubMed: 11074743]

3. Ward MM. Changes in the incidence of endstage renal disease due to lupus nephritis in the United States, 1996-2004. J Rheumatol. 2009; 36:63-67. [PubMed: 19004042]

4. Esdaile JM, Joseph L, MacKenzie T, Kashgarian M, Hayslett JP. The benefit of early treatment with immunosuppressive agents in lupus nephritis. J Rheumatol. 1994; 21:2046-2051. [PubMed: 7869308] 
5. Faurschou M, Starklint H, Halberg P, Jacobsen S. Prognostic factors in lupus nephritis: diagnostic and therapeutic delay increases the risk of terminal renal failure. J Rheumatol. 2006; 33:1563-1569. [PubMed: 16881113]

6. Oelzner P, Deliyska B, Funfstuck R, Hein G, Herrmann D, Stein G. Anti-C1q antibodies and antiendothelial cell antibodies in systemic lupus erythematosus-relationship with disease activity and renal involvement. Clin Rheumatol. 2003; 22:271-278. [PubMed: 14576989]

7. Moroni G, Radice A, Giammarresi G, et al. Are laboratory tests useful for monitoring the activity of lupus nephritis? A 6-year prospective study in a cohort of 228 patients with lupus nephritis. Ann Rheum Dis. 2009; 68:234-237. [PubMed: 18718989]

8. Esdaile JM, Joseph L, Abrahamowicz M, Li Y, Danoff D, Clarke AE. Routine immunologic tests in systemic lupus erythematosus: is there a need for more studies? J Rheumatol. 1996; 23:1891-1896. [PubMed: 8923362]

9. Illei GG, Tackey E, Lapteva L, Lipsky PE. Biomarkers in systemic lupus erythematosus. I. General overview of biomarkers and their applicability. Arthritis Rheum. 2004; 50:1709-1720. [PubMed: 15188346]

10. Liu CC, Manzi S, Ahearn JM. Biomarkers for systemic lupus erythematosus: a review and perspective. Curr Opin Rheumatol. 2005; 17:543-549. [PubMed: 16093831]

11. Illei GG, Tackey E, Lapteva L, Lipsky PE. Biomarkers in systemic lupus erythematosus: II. Markers of disease activity. Arthritis Rheum. 2004; 50:2048-2065. [PubMed: 15248202]

12. Illei GG, Lipsky PE. Biomarkers in systemic lupus erythematosus. Curr Rheumatol Rep. 2004; 6:382-390. [PubMed: 15355751]

13. Zhang $\mathrm{X}$, Jin $\mathrm{M}$, Wu H, et al. Biomarkers of lupus nephritis determined by serial urine proteomics. Kidney Int. 2008; 74:799-807. [PubMed: 18596723]

14. Balow JE. Clinical presentation and monitoring of lupus nephritis. Lupus. 2005; 14:25-30. [PubMed: 15732284]

15. Gladman DD, Ibanez D, Urowitz MB. Systemic lupus erythematosus disease activity index 2000. J Rheumatol. 2002; 29:288-291. [PubMed: 11838846]

16. Yee CS, Farewell V, Isenberg DA, et al. British Isles Lupus Assessment Group 2004 index is valid for assessment of disease activity in systemic lupus erythematosus. Arthritis Rheum. 2007; 56:4113-4119. [PubMed: 18050213]

17. Siedner MJ, Christopher-Stine L, Astor BC, Gelber AC, Fine DM. Screening for proteinuria in patients with lupus: a survey of practice preferences among American rheumatologists. J Rheumatol. 2007; 34:973-977. [PubMed: 17444588]

18. Siedner MJ, Gelber AC, Rovin BH, et al. Diagnostic accuracy study of urine dipstick in relation to 24-hour measurement as a screening tool for proteinuria in lupus nephritis. J Rheumatol. 2008; 35:84-90. [PubMed: 18085740]

19. Christopher-Stine L, Petri M, Astor BC, Fine D. Urine protein-to-creatinine ratio is a reliable measure of proteinuria in lupus nephritis. J Rheumatol. 2004; 31:1557-1559. [PubMed: 15290735]

20. Ginsberg JM, Chang BS, Matarese RA, Garella S. Use of single voided urine samples to estimate quantitative proteinuria. New Eng J Med. 1983; 309:1543-1546. [PubMed: 6656849]

21. Rodby RA, Rohde RD, Sharon Z, Pohl MA, Bain RP, Lewis EJ. The urine protein to creatinine ratio as a predictor of 24-hour urine protein excretion in type 1 diabetic patients with nephropathy. The Collaborative Study Group. Am J Kidney Dis. 1995; 26:904-909. [PubMed: 7503064]

22. Eknoyan G, Hostetter T, Bakris GL, et al. Proteinuria and other markers of chronic kidney disease: a position statement of the national kidney foundation (NKF) and the national institute of diabetes and digestive and kidney diseases (NIDDK). Am J Kidney Dis. 2003; 42:617-622. [PubMed: 14520612]

23. Levey AS, Coresh J, Balk E, et al. National Kidney Foundation practice guidelines for chronic kidney disease: evaluation, classification, and stratification. Ann Int Med. 2003; 139:137-147. [PubMed: 12859163]

24. Bauer JW, Baechler EC, Petri M, et al. Elevated serum levels of interferon-regulated chemokines are biomarkers for active human systemic lupus erythematosus. PLoS Med. 2006; 3:e491. [PubMed: 17177599] 
25. Liang MH, Schur PH, Fortin P, et al. The American College of Rheumatology response criteria for proliferative and membranous renal disease in systemic lupus erythematosus clinical trials. Arthritis Rheum. 2006; 54:421-432. [PubMed: 16453282]

26. Leung YY, Szeto CC, Tam LS, et al. Urine protein-to-creatinine ratio in an untimed urine collection is a reliable measure of proteinuria in lupus nephritis. Rheumatol. 2007; 46:649-652.

27. Birmingham DJ, Rovin BH, Shidham G, et al. Spot urine protein/creatinine ratios are unreliable estimates of $24 \mathrm{~h}$ proteinuria in most systemic lupus erythematosus nephritis flares. Kidney Int. 2007; 72:865-870. [PubMed: 17653137]

28. Hebert LA, Birmingham DJ, Shidham G, Rovin B, Nagaraja HN, Yu CY. Random spot urine protein/creatinine ratio is unreliable for estimating 24-hour proteinuria in individual systemic lupus erythematosus nephritis patients. Nephron Clin Pract. 2009; 113:c177-c182. [PubMed: 19672116]

29. Toto RD. Microalbuminuria: definition, detection, and clinical significance. J Clin Hypertens. 2004; 6:2-7.

30. Christopher-Stine L, Siedner M, Lin J, et al. Renal biopsy in lupus patients with low levels of proteinuria. J Rheumatol. 2007; 34:332-335. [PubMed: 17183619]

31. Batlle-Gualda E, Martinez AC, Guerra RA, Pascual E. Urinary albumin excretion in patients with systemic lupus erythematosus without renal disease. Ann Rheumatic Dis. 1997; 56:386-389.

32. Valente de Almeida R, Rocha de Carvalho JG, de Azevedo VF, et al. Microalbuminuria and renal morphology in the evaluation of subclinical lupus nephritis. Clin Nephrol. 1999; 52:218-229. [PubMed: 10543324]

33. Gunnarsson I, Sundelin B, Heimburger M, et al. Repeated renal biopsy in proliferative lupus nephritis-predictive role of serum C1q and albuminuria. J Rheumatol. 2002; 29:693-699. [PubMed: 11950009]

34. Birmingham DJ, Rovin BH, Shidham G, Bissell M, Nagaraja HN, Hebert LA. Relationship between albuminuria and total proteinuria in systemic lupus erythematosus nephritis: diagnostic and therapeutic implications. Clin J Am Soc Nephrol. 2008; 3:1028-1033. [PubMed: 18450925]

35. Cottiero RA, Madaio MP, Levey AS. Glomerular filtration rate and urinary albumin excretion rate in systemic lupus erythematosus. Nephron. 1995; 69:140-146. [PubMed: 7723895]

36. Fine DM, Ziegenbein M, Petri M, et al. A prospective study of protein excretion using shortinterval timed urine collections in patients with lupus nephritis. Kidney Int. 2009; 76:1284-1288. [PubMed: 19759526]

37. James P. Protein identification in the post-genome era: the rapid rise of proteomics. Q Rev Biophys. 1997; 30:279-331. [PubMed: 9634650]

38. Schaub S, Wilkins J, Weiler T, Sangster K, Rush D, Nickerson P. Urine protein profiling with surface-enhanced laser-desorption/ionization time-of-flight mass spectrometry. Kidney Int. 2004; 65:323-332. [PubMed: 14675066]

39. Wu T, Mohan C. Proteomic toolbox for autoimmunity research. Autoimmun Rev. 2009; 8:595598. [PubMed: 19393208]

40. Suzuki M, Ross GF, Wiers K, et al. Identification of a urinary proteomic signature for lupus nephritis in children. Pediatr Nephrol. 2007; 22:2047-2057. [PubMed: 17901988]

41. Suzuki M, Wiers K, Brooks EB, et al. Initial validation of a novel protein biomarker panel for active pediatric lupus nephritis. Pediatr Res. 2009; 65:530-536. [PubMed: 19218887]

42. Uehara Y, Makino H, Seiki K, Urade Y. Urinary excretions of lipocalin-type prostaglandin D synthase predict renal injury in type-2 diabetes: a cross-sectional and prospective multicentre study. Nephrol Dial Transplant. 2009; 24:475-482. [PubMed: 18799608]

43. Mosley K, Tam FW, Edwards RJ, Crozier J, Pusey CD, Lightstone L. Urinary proteomic profiles distinguish between active and inactive lupus nephritis. Rheumatol. 2006; 45:1497-1504.

44. Varghese SA, Powell TB, Budisavljevic MN, et al. Urine biomarkers predict the cause of glomerular disease. J Am Soc Nephrol. 2007; 18:913-922. [PubMed: 17301191]

45. Oates JC, Varghese S, Bland AM, et al. Prediction of urinary protein markers in lupus nephritis. Kidney Int. 2005; 68:2588-2592. [PubMed: 16316334]

46. Lee P, Peng H, Gelbart T, Wang L, Beutler E. Regulation of hepcidin transcription by interleukin-1 and interleukin-6. Proc Natl Acad Sci USA. 2005; 102:1906-1910. [PubMed: 15684062] 
47. Wu T, Fu Y, Brekken D, et al. Urine proteome scans uncover total urinary protease, PGDS, SAP and SOD as potential markers of lupus nephritis. J Immunol. 201010.4049/jimmunol.0900292

48. Dienz O, Rincon M. The effects of IL-6 on CD4 T cell responses. Clin Immunol. 2009; 130:27-33. [PubMed: 18845487]

49. Li Y, Tucci M, Narain S, et al. Urinary biomarkers in lupus nephritis. Autoimmun Rev. 2006; 5:383-388. [PubMed: 16890891]

50. Okada M, Kitahara M, Kishimoto S, Matsuda T, Hirano T, Kishimoto T. IL-6/BSF-2 functions as a killer helper factor in the in vitro induction of cytotoxic T cells. J Immunol. 1988; 141:1543-1549. [PubMed: 3261754]

51. Tanigawa T, Nicola N, McArthur GA, Strasser A, Begley CG. Differential regulation of macrophage differentiation in response to leukemia inhibitory factor/oncostatin-M/interleukin-6: the effect of enforced expression of the SCL transcription factor. Blood. 1995; 85:379-390. [PubMed: 7811994]

52. Castell JV, Gomez-Lechon MJ, David M, Hirano T, Kishimoto T, Heinrich PC. Recombinant human interleukin-6 (IL-6/BSF-2/HSF) regulates the synthesis of acute phase proteins in human hepatocytes. FEBS Lett. 1988; 232:347-350. [PubMed: 2454206]

53. Richards HB, Satoh M, Shaw M, Libert C, Poli V, Reeves WH. Interleukin 6 dependence of antiDNA antibody production: evidence for two pathways of autoantibody formation in pristaneinduced lupus. J Exp Med. 1998; 188:985-990. [PubMed: 9730900]

54. Iwano M, Dohi K, Hirata E, et al. Urinary levels of IL-6 in patients with active lupus nephritis. Clin Nephrol. 1993; 40:16-21. [PubMed: 8358870]

55. Tesar V, Masek Z, Rychlik I, et al. Cytokines and adhesion molecules in renal vasculitis and lupus nephritis. Nephrol Dial Transplant. 1998; 13:1662-1667. [PubMed: 9681708]

56. Tsai CY, Wu TH, Yu CL, Lu JY, Tsai YY. Increased excretions of beta2-microglobulin, IL-6, and IL-8 and decreased excretion of Tamm-Horsfall glycoprotein in urine of patients with active lupus nephritis. Nephron. 2000; 85:207-214. [PubMed: 10867535]

57. Alon R, Kassner PD, Carr MW, Finger EB, Hemler ME, Springer TA. The integrin VLA-4 supports tethering and rolling in flow on VCAM-1. J Cell Biol. 1995; 128:1243-1253. [PubMed: 7534768]

58. McHale JF, Harari OA, Marshall D, Haskard DO. TNF-alpha and IL-1 sequentially induce endothelial ICAM-1 and VCAM-1 expression in MRL/lpr lupus-prone mice. J Immunol. 1999; 163:3993-4000. [PubMed: 10491002]

59. Baran D, Vendeville B, Ogborn M, Katz N. Cell adhesion molecule expression in murine lupuslike nephritis induced by lipopolysaccharide. Nephron. 2000; 84:167-176. [PubMed: 10657718]

60. Wu T, Xie C, Bhaskarabhatla M, et al. Excreted urinary mediators in an animal model of experimental immune nephritis with potential pathogenic significance. Arthritis Rheum. 2007; 56:949-959. [PubMed: 17328072]

61. Wu T, Xie C, Wang HW, et al. Elevated urinary VCAM-1, P-selectin, soluble TNF receptor-1, and CXC chemokine ligand 16 in multiple murine lupus strains and human lupus nephritis. J Immunol. 2007; 179:7166-7175. [PubMed: 17982109]

62. Kiani A, Mohan C, Wu T, Madger L, Petri M. VCAM-1 is a better measure of SLE renal activity than NGAL and CXCL16. Arthritis Rheum. 2009; 60:S345.

63. Ikeda Y, Fujimoto T, Ameno M, Shiiki H, Dohi K. Relationship between lupus nephritis activity and the serum level of soluble VCAM-1. Lupus. 1998; 7:347-354. [PubMed: 9696139]

64. Gough PJ, Garton KJ, Wille PT, Rychlewski M, Dempsey PJ, Raines EW. A disintegrin and metalloproteinase 10-mediated cleavage and shedding regulates the cell surface expression of CXC chemokine ligand 16. J Immunol. 2004; 172:3678-3685. [PubMed: 15004171]

65. Shimaoka T, Nakayama T, Fukumoto N, et al. Cell surface-anchored SR-PSOX/CXC chemokine ligand 16 mediates firm adhesion of CXC chemokine receptor 6-expressing cells. J Leukoc Biol. 2004; 75:267-274. [PubMed: 14634054]

66. Minami M, Kume N, Shimaoka T, et al. Expression of scavenger receptor for phosphatidylserine and oxidized lipoprotein (SR-PSOX) in human atheroma. Ann NY Acad Sci. 2001; 947:373-376. [PubMed: 11795294] 
67. Chandrasekar B, Bysani S, Mummidi S. CXCL16 signals via Gi, phosphatidylinositol 3-kinase, Akt, I kappa B kinase, and nuclear factor-kappa B and induces cell-cell adhesion and aortic smooth muscle cell proliferation. J Biol Chem. 2004; 279:3188-3196. [PubMed: 14625285]

68. Shimaoka T, Nakayama T, Kume N, et al. Cutting edge: SR-PSOX/CXC chemokine ligand 16 mediates bacterial phagocytosis by APCs through its chemokine domain. J Immunol. 2003; 171:1647-1651. [PubMed: 12902461]

69. Teramoto K, Negoro N, Kitamoto K, et al. Microarray analysis of glomerular gene expression in murine lupus nephritis. J Pharmacol Sci. 2008; 106:56-67. [PubMed: 18187931]

70. Luster AD. Chemokines-chemotactic cytokines that mediate inflammation. N Engl J Med. 1998; 338:436-445. [PubMed: 9459648]

71. Neville LF, Mathiak G, Bagasra O. The immunobiology of interferon-gamma inducible protein 10 $\mathrm{kD}$ (IP-10): a novel, pleiotropic member of the C-X-C chemokine superfamily. Cytokine Growth Factor Rev. 1997; 8:207-219. [PubMed: 9462486]

72. Shiozawa F, Kasama T, Yajima N, et al. Enhanced expression of interferon-inducible protein 10 associated with Th1 profiles of chemokine receptor in autoimmune pulmonary inflammation of MRL/lpr mice. Arthritis Res Ther. 2004; 6:R78-R86. [PubMed: 14979941]

73. Narumi S, Takeuchi T, Kobayashi Y, Konishi K. Serum levels of ifn-inducible PROTEIN-10 relating to the activity of systemic lupus erythematosus. Cytokine. 2000; 12:1561-1565. [PubMed: 11023674]

74. Avihingsanon Y, Phumesin P, Benjachat T, et al. Measurement of urinary chemokine and growth factor messenger RNAs: a noninvasive monitoring in lupus nephritis. Kidney Int. 2006; 69:747753. [PubMed: 16518330]

75. Chicheportiche Y, Bourdon PR, Xu H, et al. TWEAK, a new secreted ligand in the tumor necrosis factor family that weakly induces apoptosis. J Biol Chem. 1997; 272:32401-32410. [PubMed: 9405449]

76. Meighan-Mantha RL, Hsu DK, Guo Y, et al. The mitogen-inducible Fn14 gene encodes a type I transmembrane protein that modulates fibroblast adhesion and migration. J Biol Chem. 1999; 274:33166-33176. [PubMed: 10551889]

77. Maecker H, Varfolomeev E, Kischkel F, et al. TWEAK attenuates the transition from innate to adaptive immunity. Cell. 2005; 123:931-944. [PubMed: 16325585]

78. Wiley SR, Cassiano L, Lofton T, et al. A novel TNF receptor family member binds TWEAK and is implicated in angiogenesis. Immunity. 2001; 15:837-846. [PubMed: 11728344]

79. Feng SL, Guo Y, Factor VM, et al. The Fn14 immediate-early response gene is induced during liver regeneration and highly expressed in both human and murine hepatocellular carcinomas. Am J Pathol. 2000; 156:1253-1261. [PubMed: 10751351]

80. Campbell S, Michaelson J, Burkly L, Putterman C. The role of TWEAK/Fn14 in the pathogenesis of inflammation and systemic autoimmunity. Front Biosci. 2004; 9:2273-2284. [PubMed: 15353286]

81. Campbell S, Burkly LC, Gao HX, et al. Proinflammatory effects of TWEAK/Fn14 interactions in glomerular mesangial cells. J Immunol. 2006; 176:1889-1898. [PubMed: 16424220]

82. Gao HX, Campbell SR, Burkly LC, et al. TNF-like weak inducer of apoptosis (TWEAK) induces inflammatory and proliferative effects in human kidney cells. Cytokine. 2009; 46:24-35. [PubMed: 19233685]

83. Molano A, Lakhani P, Aran A, Burkly LC, Michaelson JS, Putterman C. TWEAK stimulation of kidney resident cells in the pathogenesis of graft versus host induced lupus nephritis. Immunol Lett. 2009; 125:119-128. [PubMed: 19573558]

84. Morris SC, Cohen PL, Eisenberg RA. Experimental induction of systemic lupus erythematosus by recognition of foreign Ia. Clin Immunol Immunopathol. 1990; 57:263-273. [PubMed: 2208807]

85. Reap EA, Sobel ES, Jennette JC, Cohen PL, Eisenberg RA. Conventional B cells, not B1 cells, are the source of autoantibodies in chronic graft-versus-host disease. J Immunol. 1993; 151:73167323. [PubMed: 7505027]

86. Zhao Z, Burkly LC, Campbell S, et al. TWEAK/Fn14 interactions are instrumental in the pathogenesis of nephritis in the chronic graft-versus-host model of systemic lupus erythematosus. J Immunol. 2007; 179:7949-7958. [PubMed: 18025243] 
87. Kaplan MJ, Ray D, Mo RR, Yung RL, Richardson BC. TRAIL (Apo2 ligand) and TWEAK (Apo3 ligand) mediate CD4+ T cell killing of antigen-presenting macrophages. J Immunol. 2000; 164:2897-2904. [PubMed: 10706675]

88. Kaplan MJ, Lewis EE, Shelden EA, et al. The apoptotic ligands TRAIL, TWEAK, and Fas ligand mediate monocyte death induced by autologous lupus T cells. J Immunol. 2002; 169:6020-6029. [PubMed: 12421989]

89. Schwartz N, Su L, Burkly LC, et al. Urinary TWEAK and the activity of lupus nephritis. J Autoimmun. 2006; 27:242-250. [PubMed: 17257812]

90. Schwartz N, Rubinstein T, Burkly LC, et al. Urinary TWEAK as a biomarker of lupus nephritis: a multicenter cohort study. Arthritis Res Ther. 2009; 11:R143. [PubMed: 19785730]

91. Rovin BH, Zhang X. Biomarkers for lupus nephritis: the quest continues. Clin J Am Soc Nephrol. 2009; 4:1858-1865. [PubMed: 19729426]

92. Schwartz N, Michaelson JS, Putterman C. Lipocalin-2, TWEAK, and other cytokines as urinary biomarkers for lupus nephritis. Ann NY Acad Sci. 2007; 1109:265-274. [PubMed: 17785315]

93. Das L, Suzuki M, Devarajan P, Rovin B, Ying J, Brunner HI. Candidate urinary biomarkers may predict histological features on lupus nephritis biopsy. Arthritis Rheum. 2009; 60:S750. 


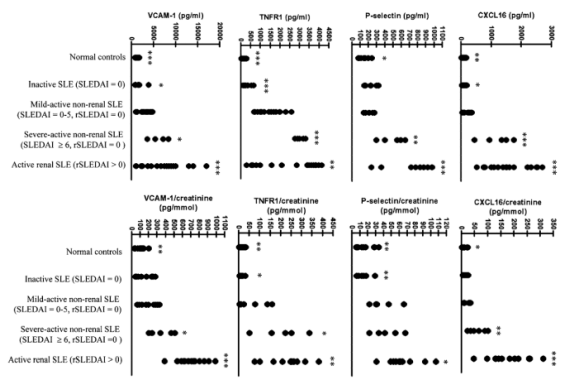

Fig. 1.

Reproduced with permission from the Journal of Immunology, Copyright 2007. The American Association of Immunologists, Inc. [61]. Patients with active renal systemic lupus erythematosus (SLE) have significantly increased concentrations of urinary vascular cell adhesion molecule-1, CXCL16, tumor necrosis factor receptor-1, and P-selectin. Indicated $p$ values pertain to Student's $t$ test or Mann-Whitney $U$ test comparisons of each subject group vs. mild-active, non-renal SLE $(* p<0.05, * * p<0.01, * * * p<0.001)$ 


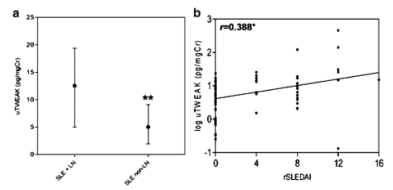

Fig. 2.

Reproduced with permission from Arthritis Research and Therapy [90]. Urinary TWEAK levels are significantly lower in systemic lupus erythematosus patients without lupus nephritis (a), and the log of urinary TWEAK correlates with increasing Systemic Lupus Erythematosus Disease Activity Index scores (b). ${ }^{*} p<0.05$ 


\section{Table 1}

Performance of historical biomarkers [6-8]

\begin{tabular}{llc}
\hline Test & Sensitivity & Specificity \\
\hline Anti-dsDNA & $53-100 \%$ & $50-69 \%$ \\
C3 & $56-79 \%$ & $51-64 \%$ \\
C4 & $53-74 \%$ & $64-65 \%$ \\
Anti-C1q & $53-81 \%$ & $64-71 \%$ \\
\hline
\end{tabular}

Sensitivity and specificity ranges of historical biomarkers for active lupus nephritis 
Table 2

Methods for analysis of urinary protein

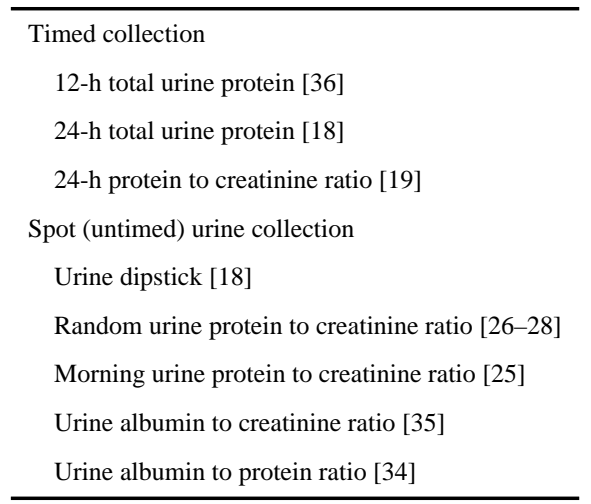

Multiple methods have been described for the clinical assessment of urinary protein in lupus patients, including both timed and spot sample collection techniques. Further detail is provided in the text 


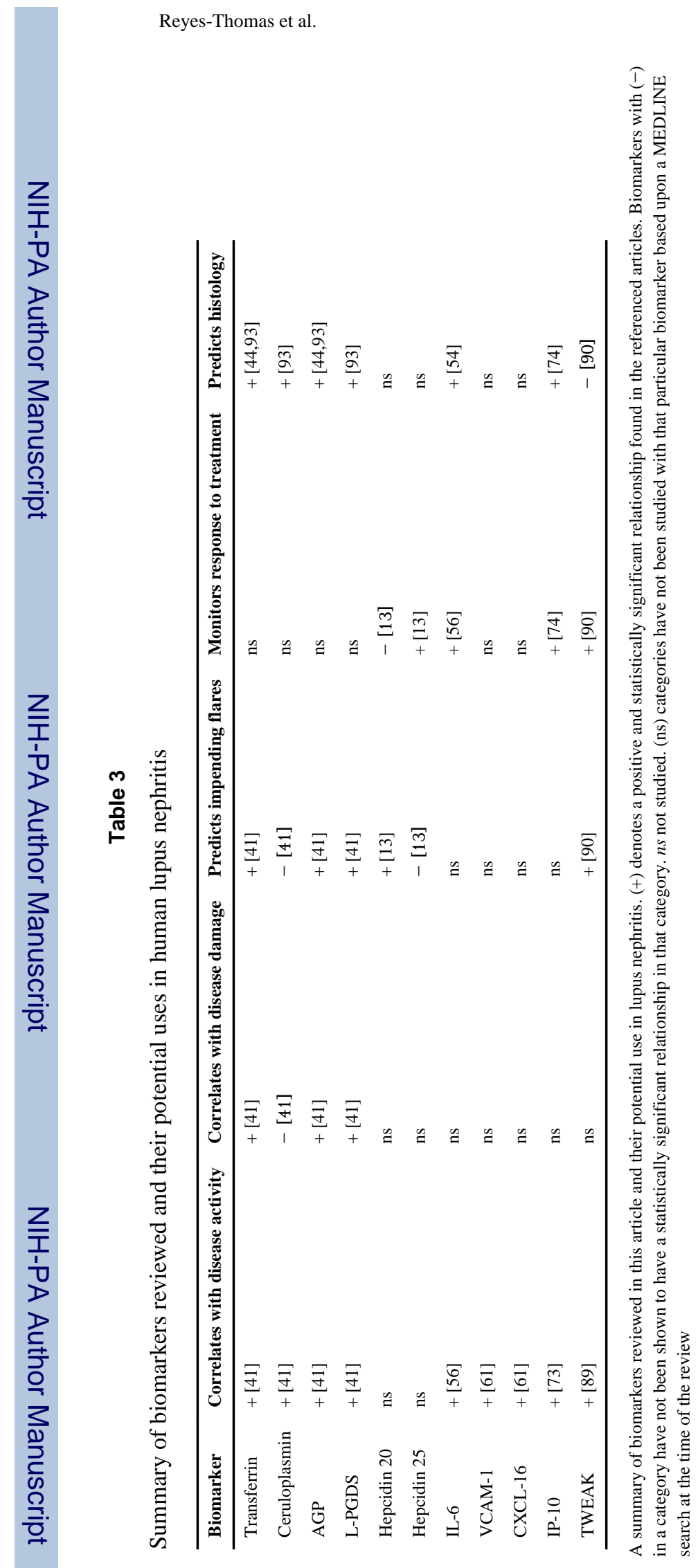

Clin Rev Allergy Immunol. Author manuscript; available in PMC 2012 June 1. 